\title{
Maternal mortality and morbidity burden in the Eastern Mediterranean Region: findings from the Global Burden of Disease 2015 study
}

\author{
GBD 2015 Eastern Mediterranean Region Maternal Mortality Collaborators ${ }^{1}$
}

Received: 1 May 2017/Revised: 15 June 2017/Accepted: 21 June 2017/Published online: 3 August 2017

(c) The Author(s) 2017. This article is an open access publication

\begin{abstract}
Objectives Assessing the burden of maternal mortality is important for tracking progress and identifying public health gaps. This paper provides an overview of the burden of maternal mortality in the Eastern Mediterranean Region (EMR) by underlying cause and age from 1990 to 2015 .

Methods We used the results of the Global Burden of Disease 2015 study to explore maternal mortality in the EMR countries.

Results The maternal mortality ratio in the EMR decreased $16.3 \%$ from 283 (241-328) maternal deaths per 100,000 live births in 1990 to 237 (188-293) in 2015. Maternal mortality ratio was strongly correlated with socio-demographic status, where the lowest-income countries contributed the most to the burden of maternal mortality in the region.

Conclusion Progress in reducing maternal mortality in the EMR has accelerated in the past 15 years, but the burden remains high. Coordinated and rigorous efforts are needed
\end{abstract}

This article is part of the supplement "The state of health in the Eastern Mediterranean Region, 1990-2015".

The members of GBD (Global Burden of Disease) 2015 Eastern Mediterranean Region Maternal Mortality Collaborators are listed at the end of the article. Ali H. Mokdad, on behalf of GBD 2015 Eastern Mediterranean Region Maternal Mortality Collaborators, is the corresponding author.

Electronic supplementary material The online version of this article (doi:10.1007/s00038-017-1004-3) contains supplementary material, which is available to authorized users.

GBD 2015 Eastern Mediterranean Region Maternal Mortality Collaborators

mokdaa@uw.edu

1 Institute for Health Metrics and Evaluation, University of Washington, Seattle, WA, USA to make sure that adequate and timely services and interventions are available for women at each stage of reproductive life.

Keywords Maternal mortality $\cdot$ Maternal health $\cdot$ Eastern Mediterranean Region · Burden of disease

\section{Introduction}

Maternal mortality ratio (MMR), which measures deaths per 100,000 live births, is a standard measure for global, regional, and national comparison (Abouzahir and Wardlaw 2001). It is also one of the main criteria of health outcomes and an indicator of the socioeconomic development level of countries that is recognized worldwide (Liang et al. 2010). Country estimates of maternal mortality over time are crucial to inform the planning of maternal, sexual, and reproductive health programs and to guide advocacy efforts and research at the national level. These estimates are also needed at the international level to inform decision-making concerning resource allocation by development partners and donors (WHO et al. 2007).

In 2015, 275,288 women are estimated to have died as a result of pregnancy or childbirth globally (Kassebaum et al. 2016b). Maternal death is defined by ICD-10 as the death of a woman while pregnant or within 42 days and up to 1 year (late maternal death); of termination of pregnancy, irrespective of the duration and site of the pregnancy, from any cause related to or aggravated by the pregnancy or its management, but not from accidental or incidental causes (World Health Organization 1992).

The primary causes of maternal deaths are hemorrhage, hypertension, infections, pre-existing medical conditions, and lack of literacy, family planning (unmet need and birth 
spacing), and access to pregnancy termination when needed. Identifying and improving the main factors related to maternal mortality depend on a correct definition of the required priorities for appropriate prevention, diagnosis, and treatment (Ribeiro et al. 2008).

Poverty, illiteracy, malnutrition, and the low social status of women are usually among the major underlying causes of maternal mortality. Analyzing the economic, social, and health system factors affecting maternal mortality can provide some credible information and evidence for public health interventions to improve maternal health. A few general studies on nonclinical determinants of maternal mortality have analyzed different factors including access to safe drinking water, access to food, fertility rate, education level, life expectancy at birth, access to health services, delivery done by skilled birth attendants, out-of-pocket payment, GDP per capita, health expenditures, and government corruption (Hertz et al. 1994; Midhet et al. 1998; Buor and Bream 2004; Alvarez et al. 2009; Muldoon et al. 2011).

From 1990 to 2015, the global MMR decreased 30.6\% from 280.4 (95\% uncertainty interval (UI): 262.6-299.0) to 194.7 (172.6-233.2) (Institute for Health Metrics and Evaluation 2017). This decrease was driven in part by the recognition of maternal health as one of the priorities in the UN Millennium Development Goals (MDGs), which provided a strong incentive to reduce MMR by country (Ronsmans and Graham 2006). New goals outlined in the sustainable development goals (SDGs) for 2015 to 2030 build on the momentum of the MDGs and seek to bring the global MMR below 70 deaths per 100,000 live births (United Nations 2015). The greatest challenge in meeting this goal lies with countries in the bottom two quintiles of wealth, where improvement is most needed and has historically occurred slowly if at all (Abouzahir and Wardlaw 2001).

The variation in MMR between countries by income group is drastic, where the burden of maternal mortality falls almost exclusively upon developing countries (Ronsmans and Graham 2006). Globally, the average MMR for countries in the top quintile of wealth for 2015 was 14.5 (13.7-15.4) compared to 440.2 (357.4-543.5) in the bottom quintile (Institute for Health Metrics and Evaluation 2017). Despite this variation, inequalities in maternal mortality exist between and within countries, where mortality in all locations is exacerbated by a lack of access to quality obstetric care, whether because of the remoteness of certain regions or the inability of health facilities to provide proper care (Peterson et al. 2012).

The Eastern Mediterranean Region (EMR) is a diverse region consisting of 22 countries, including high-income countries, where socioeconomic development has progressed considerably over the last decades; middle-income countries that have well-developed health service delivery infrastructures but face resource constraints; and low-income countries that lack the resources and infrastructure for effective health interventions. Many countries in the region are suffering from political instability, conflicts, and other complex development challenges. This range from low to high incomes can be seen as a microcosm of the variability in maternal mortality present globally. This paper seeks to examine in detail the burden of maternal mortality within the EMR using the results of the GBD 2015 study.

\section{Methods}

A detailed methodology of maternal mortality estimation for GBD 2015 has been published elsewhere (Kassebaum et al. 2016b). In short, sources with population-level data were processed using standardized algorithms to adjust for age-specific, year-specific, and geography-specific patterns of incompleteness. Additional steps were taken to account for underestimations of maternal mortality in vital registration systems. ICD-10 vital registration codes pertaining to HIV-related maternal deaths were excluded and quantified separately (Kassebaum et al. 2014). Overall maternal mortality was modeled using cause-of-death ensemble modeling (CODEm), where all combinations of covariates were tested and ranked on the basis of out-of-sample predictive validity performance. All data sources are available on our website, and we provide a visualization to show each source used in our analyses (Institute for Health Metrics and Evaluation 2016).

We calculated years of life lost (YLLs) by multiplying deaths by the residual expected individual lifespan at the age of death as derived from the GBD 2015 standard model life table. Years lived with disability (YLDs) were calculated by multiplying the number of prevalent cases of a certain health outcome by the disability weight assigned to this health outcome. A disability weight reflects the magnitude of the health loss associated with an outcome and has a value that is anchored between 0 , equivalent to full health, and 1, equivalent to death. YLLs were calculated by multiplying deaths by the remaining life expectancy at the age of death from a standard life table chosen as the norm for estimating premature mortality in GBD. Disability-adjusted life years (DALYs) were calculated by adding YLLs and YLDs. Detailed methods on YLLs, YLDs, and DALYs are published elsewhere (Kassebaum et al. 2016a; Vos et al. 2016; Wang et al. 2016).

We evaluated the associations between maternal mortality and socio-demographic status using the socio-demographic index (SDI). SDI is a composite measure developed for GBD 2015 that accounts for fertility rate, lag-dependent income per capita, and education. To capture the average relationships for each age group, we applied a simple least squares spline 
regression of the maternal mortality rate on SDI. The SDI is scaled from 0 to 1 , where 0 represents the lowest possible observed SDI and 1 is the highest. We reported uncertainty for all our estimates (Kassebaum et al. 2016a).

\section{Results}

\section{Mortality}

The total number of deaths due to maternal disorders increased $4.5 \%$ from $38,595(32,859-44,785)$ deaths in 1990 to $40,338(31,965-49,954)$ in 2015 in the EMR, compared to a $29.5 \%$ decrease globally (Institute for Health Metrics and Evaluation 2017). In 1990, the maternal mortality ratio was similar in the EMR and globally at 283 (241-328) maternal deaths per 100,000 live births in the EMR and 280 (263-299) globally (Institute for Health Metrics and Evaluation 2017). By 1995, a gap between the regional and global trends had opened, and this persisted steadily through 2015 . By then, the EMR had a maternal mortality ratio of 237 (188-293) compared to 194 (173-223) globally (Institute for Health Metrics and Evaluation 2017). By country, only Afghanistan and Djibouti had an annual increase in maternal mortality ratio between 1990 and 2015 (Table 1). Palestine also had an increase in maternal mortality ratio between 2000 and 2015, but still had a lower ratio in 2015 compared to 1990 (Table 1).

Both globally and regionally, maternal hemorrhage was the leading cause of maternal mortality in 1990 and 2015 (Institute for Health Metrics and Evaluation 2017). By country within the EMR, hemorrhage was also the most common, followed by other maternal disorders, noticeably within the Gulf states (Fig. 1). In all countries, all causes of maternal mortality decreased from 1990 to 2015 (Table 2). In 2015, maternal hemorrhage in Somalia was the largest contributor to maternal mortality by country and causes at 72.3 (13.2-229.0) deaths per 100,000 among women aged 15-49 (Table 2). The GBD study expanded the analysis to the full reproductive age range of 10-54 years (Kassebaum et al. 2016b). In general, the countries with the highest mortality rates by cause also showed the least improvement from 1990 to 2015 (Table 2).

\section{YLLS}

YLL rates fell 49\% in the EMR between 1990 and 2015 , the same as the global mean, from 2,683 (2284-3103) per 100,000 women aged 15-49 to 1377 (1091-1695) (Table 3). In that time frame, the YLL rate decreased in all countries (Table 3). There was significant inter-country variation in YLL rates. The highest YLL rate in 2015 was in Somalia with 7774 (1497-23,408) YLLs per 100,000 women aged 15-49, compared to Kuwait at 21 (16-27) (Table 3). Somalia also had the smallest percent change from 1990 to 2015, a decrease of $18 \%$ (Table 3).

\section{YLDs}

Average YLD rates in the region fell from 161 (114-215) per 100,000 women aged $15-49$ to 82 (57-113), a $49 \%$ decrease (Table 3). Globally, there was a $41 \%$ decrease (Institute for Health Metrics and Evaluation 2017). Inter-country variability in the YLD rate was less than that of YLLs, with the highest seen in Somalia with 254 (171-362) YLDs per 100,000 women aged 15-49 and the lowest in the United Arab Emirates with 16 (10-26) (Table 3).

\section{YLL/YLD}

The YLL/YLD ratio was included as an indicator of health system effectiveness in dealing with fatal and non-fatal outcomes of maternal disorders. Large values, such as that of Afghanistan, a ratio of 89.6 in 2015, demonstrated cases, where fatal outcomes heavily outweigh non-fatal ones (Table 3). Kuwait in 2015 had a ratio of 1.11, signifying a near equal burden of fatal and non-fatal outcomes for maternal disorders (Table 3). Lower income countries with SDI less than 0.5 all had ratios greater than 10 , whereas higher income countries with SDI greater than 0.7 had ratios under 4 (Table 3). In the countries with SDI less than 0.5 , all but Sudan had an increase in YLL/YLD ratio from 1990 to 2015 (Table 3). Large decreases in the ratio from 1990 to 2015 highlighted effective improvements in treatment, such as in Morocco, where the ratio decreased from 35.2 to 8.5 (Table 3).

\section{DALYs}

From 1990 to 2015, DALY rates decreased in all countries in the region. The overall DALY rate for the region decreased by $49 \%$ during this interval, compared to $51 \%$ globally (Institute for Health Metrics and Evaluation 2017). As discussed in the YLL/YLD section above, the proportional contribution of YLDs to the DALYs rate increased as SDI increased (Table 3). Like YLL rates, DALY rates showed large inter-country variation, as shown in Fig. 2. DALY rates peaked in the 20-25-year age group, decreasing until ages 35-40, then steeply falling off in 5-year increments (Fig. 3). By sub-cause, complication of abortions contributed the most to the DALY rate in young (10-15) and old (45+) age groups (e-Fig. 1). Percent of DALYs attributable to obstructed labor increased steadily with age, spiking drastically for the 50-55-year age group (e-Fig. 1). 


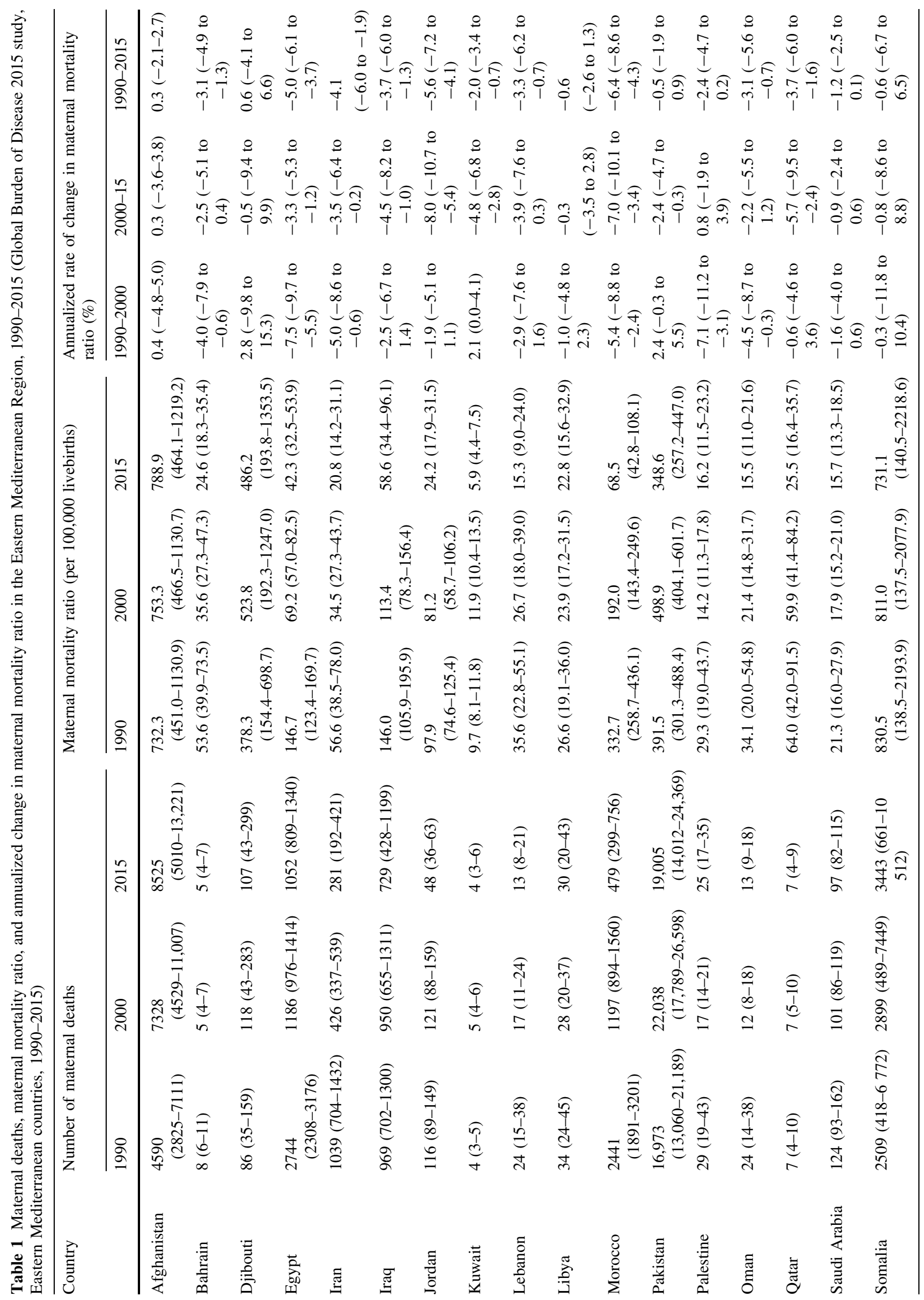




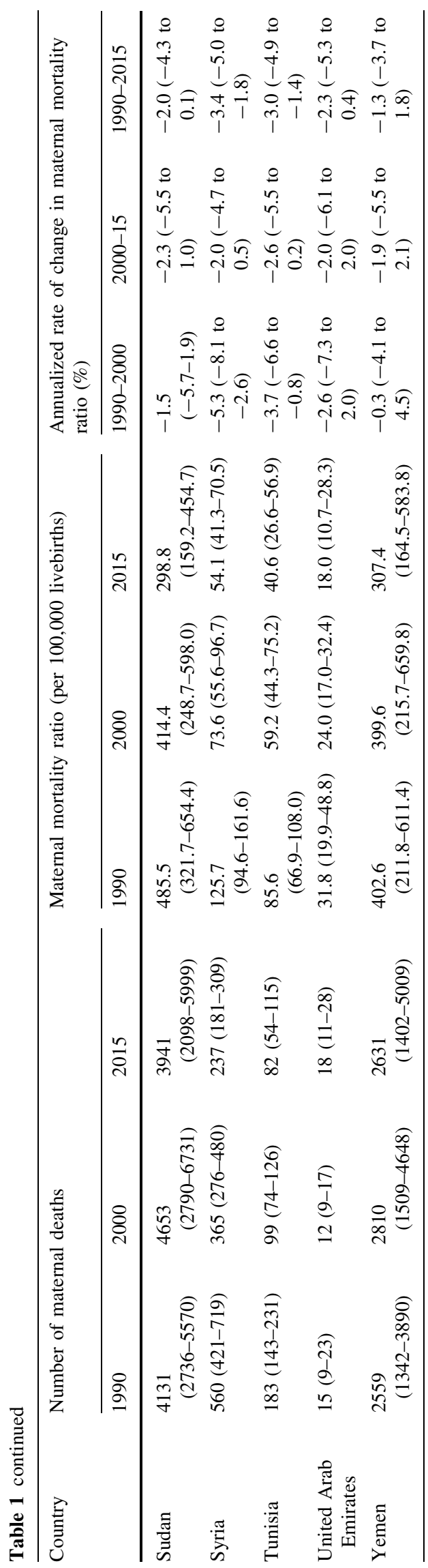

\section{Discussion}

Our study showed that maternal mortality has declined in the EMR except in Djibouti, Palestine, and Afghanistan in recent years. However, the burden is still higher than the global average. Our findings call for increased efforts to reduce maternal mortality in the region. Moreover, with unrest in the region, there is a risk of losing some of the observed gains.

Efforts to reduce maternal mortality in the EMR have varied in scope, approach, and success by country. This has resulted in great variations and disparities in maternal mortality levels between countries. The high-income countries in the region (Kuwait, Oman, Qatar, Saudi Arabia, and United Arab Emirates) have achieved reductions between 25 and 50\% compared to levels in 1990. Maternal mortality ratios in these countries ranged from 6 to 26 deaths per 100,000 live births, consistent with countries of similar income around the world. Certain middle-income countries such as Morocco and Jordan have successfully implemented interventions that have drastically reduced maternal mortality by greater than 75\% from 1990 levels. Low-income countries have seen the least improvement, or in some cases none at all. Reduction in maternal mortality in Pakistan, Somalia, Sudan, and Yemen ranged from 11 to $39 \%$, and maternal mortality in Afghanistan and Djibouti increased from 1990 to 2015. Maternal mortality ratios for these countries ranged from 299 deaths per 100,000 live births in Sudan to 789 in Afghanistan. This discussion will examine the obstacles to effective maternal care, the direct and underlying causes of maternal mortality, and the challenges faced by countries in the region to improve maternal health.

The process of improving maternal health in the region faces many challenges which have slowed progress in recent years. For many years, the EMR has been devastated by manmade disasters and conflicts, which have destroyed infrastructure in several nations and have tremendously affected the health of the population, especially for vulnerable groups such as mothers and children. The humanitarian community has made several efforts to address these challenges, and these have reduced the potential impact of the unrest. However, the disruption of health care systems has had a negative impact. The social and health impacts of political instability, domestic crises, and economic sanctions are well-documented in the region (Mokdad et al. 2016). Recent distress in the region will result in deteriorating health conditions in these countries for many years to come. Despite this, however, the region has experienced improved health and life expectancy.

The region is in need of efforts to improve preventive strategies for reducing maternal mortality. A literature 
Fig. 1 Top cause of maternal deaths in Eastern Mediterranean Region countries, 2015 (Global Burden of Disease 2015 study, Eastern Mediterranean Countries 2015)

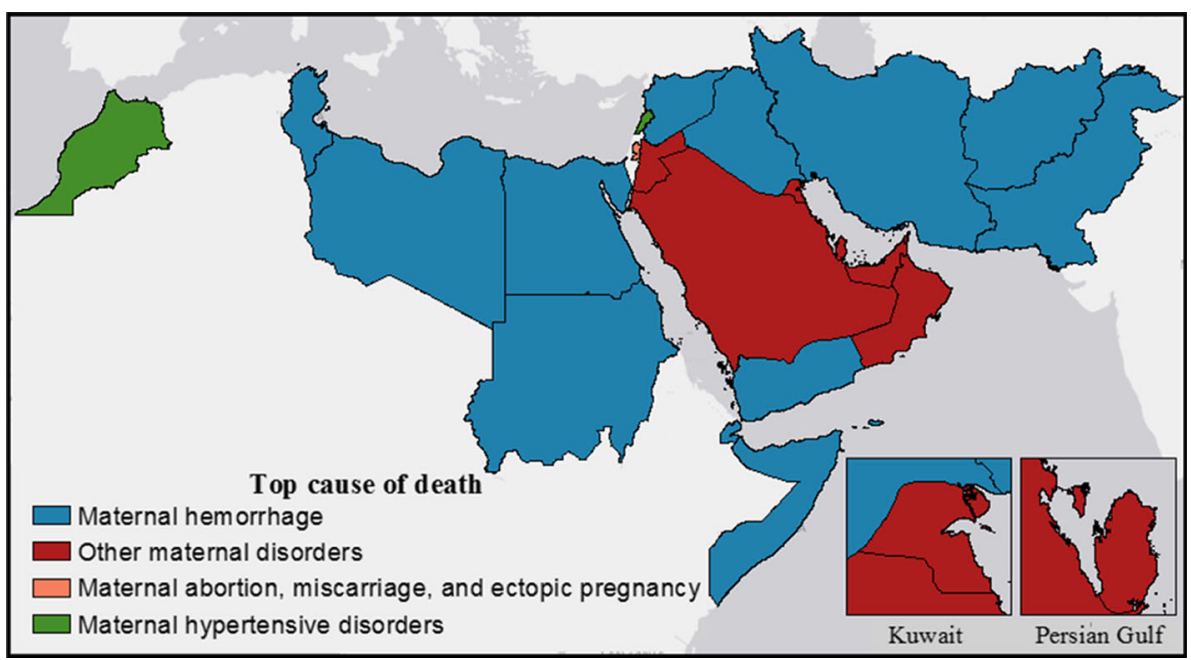

review (Warren et al. 2015) that evaluated the evidence on the effectiveness of sexual and reproductive health interventions delivered in humanitarian crises found some evidence to support increased access and demand creation for family planning services through community health workers, health care subsidies, and discussions within literacy groups. Involving communities in maternal and child health and birth preparedness programs, as well as refurbishing clinics and hospital facilities, was also associated with increased positive health outcomes (Warren et al. 2015).

Female genital mutilation (FMG) is still practiced in many countries in the EMR due to a mix of sociocultural factors within families and communities (UNICEF 2016). Experience of FGM increases the short- and long-term health risks to women and young girls between infancy and age 15 , and is unacceptable from a human rights and health perspective. Procedures can cause severe bleeding and problems urinating, and later, cysts, infections, as well as complications in childbirth and increased risk of newborn deaths (WHO 2017).

Thaddeus and Maine provide a conceptual model for understanding factors that obstruct favorable obstetric outcomes that consists of three types of delays a pregnant woman faces when seeking care-delays in deciding to seek care, in reaching an adequate facility, and in receiving adequate care in a facility. The first delay manifests itself in the way women and their families perceive the accessibility of services, often shaped by prior experiences with health facilities. Any number of factors may influence a decision to delay treatment, such as cost, distance, lack of proper medical supplies, and unhelpful or impolite staff. The second delay accounts for the time taken to reach a medical facility. This is a particularly large obstacle for rural areas in which the availability of transportation is uncertain or expensive. In addition, data on maternal deaths that occur on the way to a health facility are scarce. The third and final delays are in receiving adequate care at health facility, which can be attributed to insufficient staff, drugs, and equipment. It is necessary for interventions to effectively address the underlying causes of these delays on a countryby-country basis (Thaddeus and Maine 1994).

This conceptual model is backed by an extensive body of research on the direct and underlying causes of maternal mortality. Improving women's literacy and knowledge can significantly contribute to their health. Alvarez et al. reported a significant negative relationship between MMR and gross domestic product (GDP) per capita, health expenditure (HE), women's literacy rate, and the number of deliveries by skilled birth attendants in the study region in Africa (Alvarez et al. 2009). Another study in sub-Saharan African countries, concluded that birth in the presence of health professionals and the life expectancy at birth are highly correlated to maternal mortality. Furthermore, a convincing relationship was found between GDP per capita and health expenditure per capita and maternal mortality (Buor and Bream 2004). Similarly, a study in Pakistan reported that a significant relationship was observed between having access to health services, professional health staff, and health care during pregnancy and reductions in MMR (Midhet et al. 1998). The positive impacts of GDP, HE, and female education level on health outcomes were also reported in other studies in the EMR (Bayati et al. 2013; Homaie Rad et al. 2013).

Success stories do exist in the region: for example, Morocco was one of only ten countries globally that met the MDG 5 requirement of an annual rate of decline exceeding $5.5 \%$ for maternal mortality ratio every year from 1990 to 2015 (Kassebaum et al. 2016b). This improvement can be traced to a number of programs 


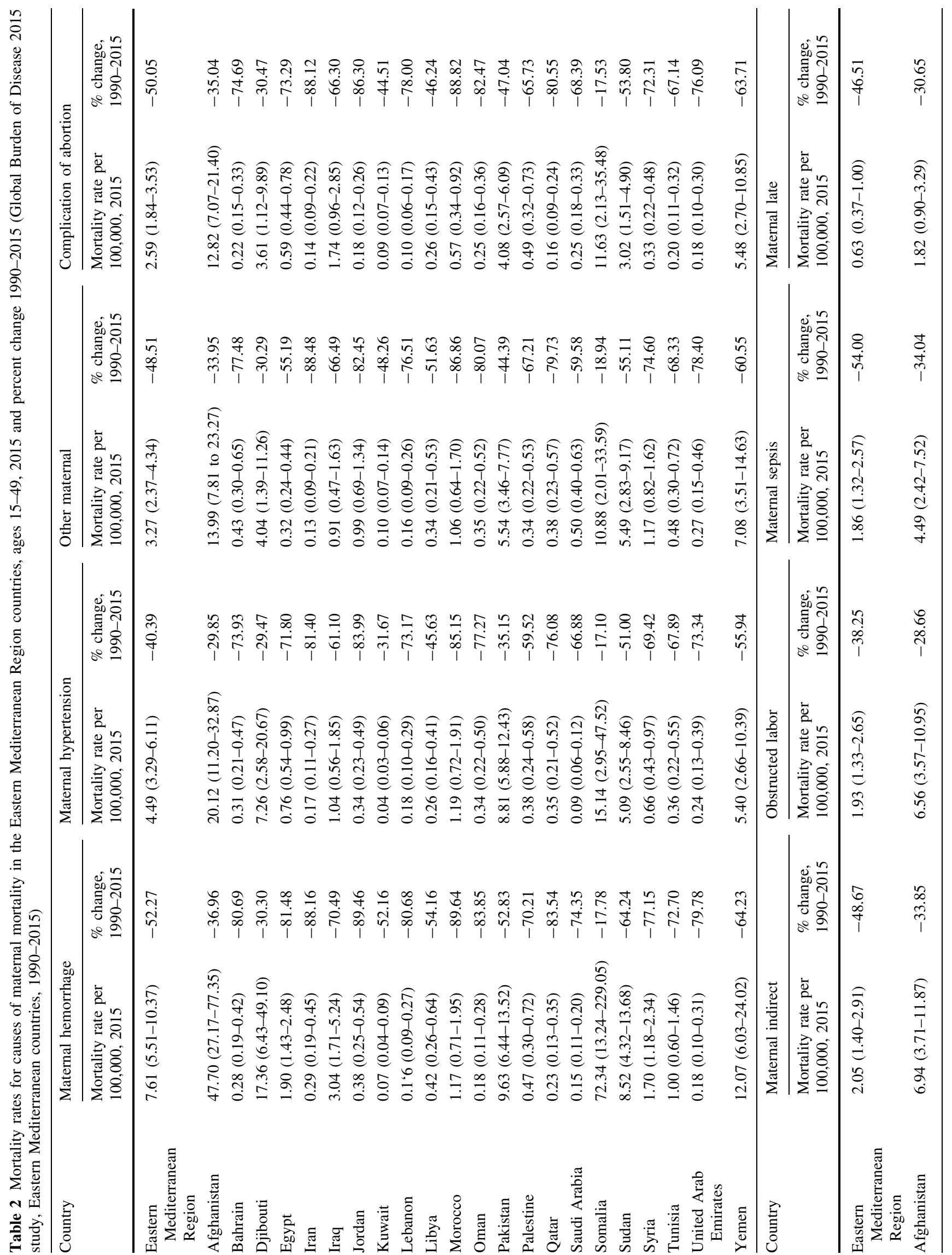




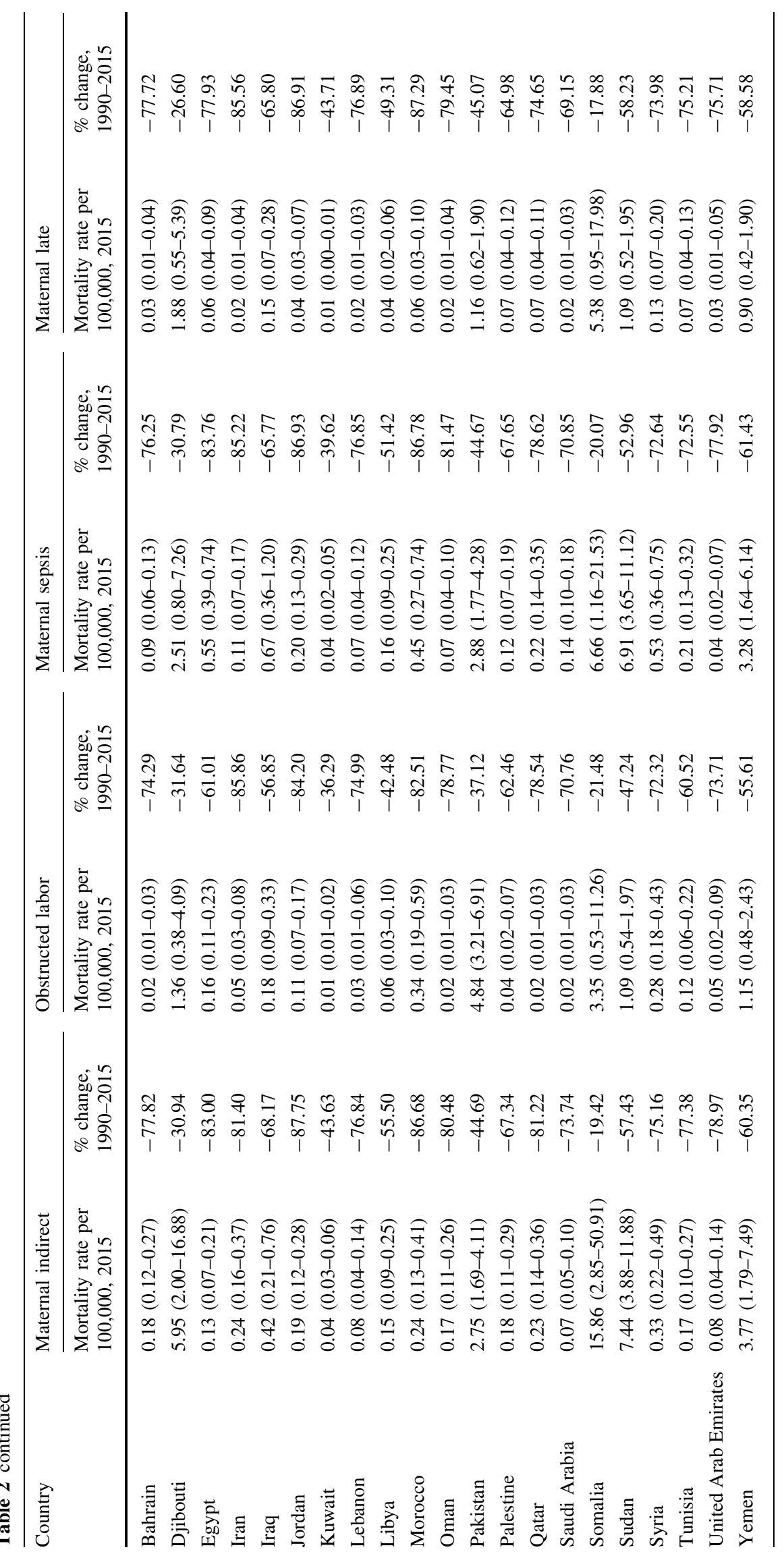




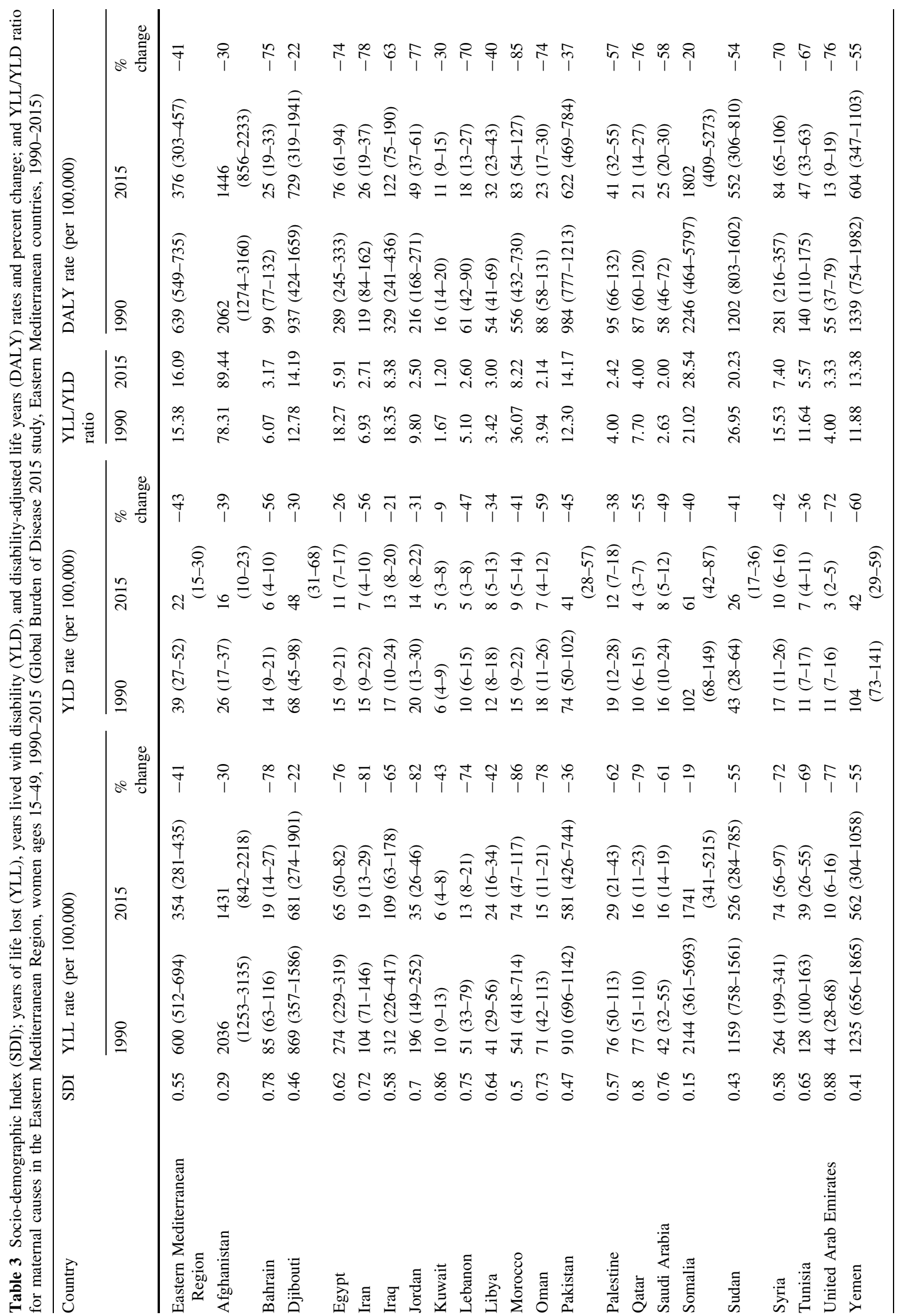


Fig. 2 Disability-adjusted lifeyear (DALY) rates per 100,000 women ages 15-49 for maternal causes by country, 2015 (Global Burden of Disease 2015 Study,

Eastern Mediterranean

Countries 2015)
Fig. 3 Disability-adjusted lifeyear (DALY) rates per 100,000 women ages $10-54$ for maternal causes in the Eastern Mediterranean Region by age, 2015 (Global Burden of Disease 2015 study, Eastern Mediterranean Region 2015)
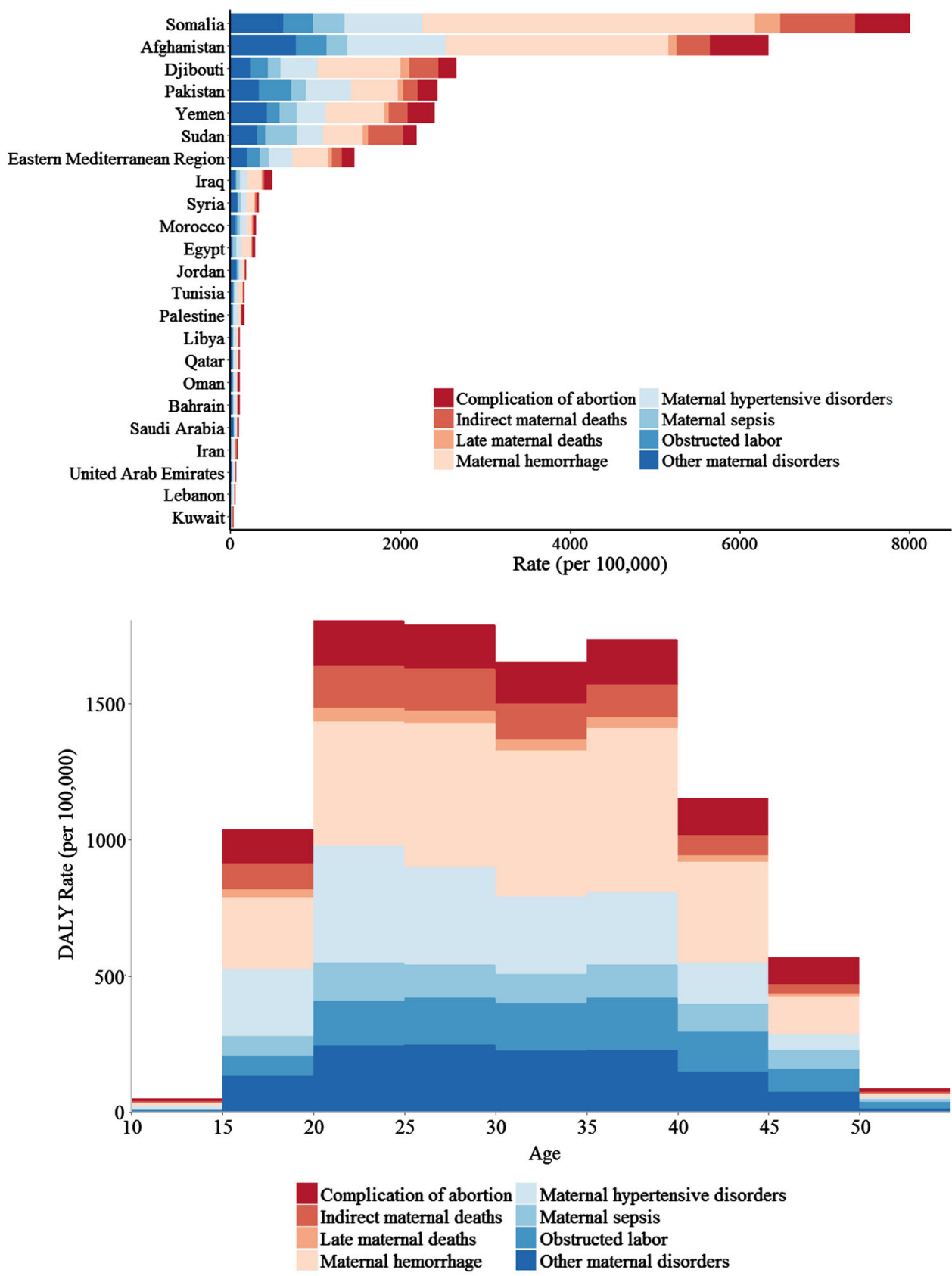

implemented to improve determinants of health and health coverage. Increases in the number of health facilities and training programs led to the percentage of deliveries assisted by skilled personnel increasing from $31 \%$ in 1992 to $73.5 \%$ in 2011 . Other successes in the region occurred in Jordan and Iran, where maternal mortality was reduced by more than 50\% from levels in 1990 (Kassebaum et al. 2016b).

Uneven improvements in MMR in the region may be related to differing levels of ramp-up in coverage for specific types of reproductive health care-antenatal care, in-facility delivery, skilled birth attendance, family planning services, emergency obstetric care, and post-natal care-that are all known to decrease the risk of negative pregnancy outcomes (Lim et al. 2010; Randive et al. 2014). Quality of care must also be considered during buildup of the maternal health care system. Programs for reproductive health care must ensure that women are receiving the care they need during pregnancy and the post-partum period (Rowe et al. 2005). Care should be integrated and not be focused on single vertical interventions (Campbell et al. 2006).

It has been challenging for many countries to produce timely and accurate data on levels of maternal mortality that would indicate the extent of their progress in reducing maternal deaths (Mokdad et al. 2016). Reliable information 
is a necessary component of any strategy aimed at reducing maternal mortality. Continued progress in data collection in the EMR is key to evaluating progress in reducing maternal mortality.

Our study has several limitations. First, many countries in the region have poor health data and vital statistics. We used GBD methodology to account for quality and lack of data. We also applied our standard GBD garbage code correction to address this limitation. Second, little information is available on unsafe abortion in the region due to religion and culture. However, our study is the most comprehensive on burden of diseases and applies the standard methodology that allows global comparison.

\section{Conclusion}

Progress in reducing maternal mortality in the EMR has accelerated in the past 15 years, but there is still much to do to reduce preventable deaths. Extending basic maternal health services, improving quality of care, and eliminating unmet need for contraception are all crucial, proven steps effective at reducing MMR. Our study showed the importance of empowering women: increased women's rights are needed to improve their health. Finally, coordinated and rigorous efforts are needed to make sure that every woman in need receives these interventions in a timely fashion at each stage of her reproductive life.

GBD 2015 Eastern Mediterranean Region Maternal Mortality Collaborators: Ali H. Mokdad, PhD (corresponding author), Institute for Health Metrics and Evaluation, University of Washington, Seattle, Washington, United States. Ibrahim Khalil, MD, Institute for Health Metrics and Evaluation, University of Washington. Michael Collison, BS, Institute for Health Metrics and Evaluation, University of Washington, Seattle, Washington, United States. Charbel El Bcheraoui, PhD, Institute for Health Metrics and Evaluation, University of Washington. Raghid Charara, MD, American University of Beirut, Beirut, Lebanon. Maziar Moradi-Lakeh, MD, Department of Community Medicine, Preventative Medicine and Public Health Research Center, Gastrointestinal and Liver Disease Research Center (GILDRC), University of Medical Sciences, Tehran, Iran. Ashkan Afshin MD, Institute for Health Metrics and Evaluation, University of Washington, Seattle, Washington, United States. Adrienne Chew, ND, Institute for Health Metrics and Evaluation, University of Washington. Farah Daoud, BA/BS, Institute for Health Metrics and Evaluation, University of Washington. Kristopher J. Krohn, BA, Institute for Health Metrics and Evaluation, University of Washington, Seattle, Washington, United States. Danny Colombara, $\mathrm{PhD}$, Institute for Health Metrics and Evaluation, University of Washington, Seattle, Washington, United States. Rebecca Ehrenkranz, MPH, Institute for Health Metrics and Evaluation, University of Washington, Seattle, Washington, United States. Michael Kutz, BS, Institute for Health Metrics and Evaluation, University of Washington, Seattle, Washington, United States. Haidong Wang, PhD, Institute for Health Metrics and Evaluation, University of Washington, Seattle, Washington, United States. Amanuel Alemu Abajobir, MPH, School of Public Health, University of Queensland, Brisbane, QLD,
Australia. Foad Abd-Allah, MD, Department of Neurology, Cairo University, Cairo, Egypt. Haftom Niguse Abraha, MS, Mekelle University, Mekelle, Ethiopia. Laith J. Abu-Raddad, PhD, Infectious Disease Epidemiology Group, Weill Cornell Medical College in Qatar, Doha, Qatar. Aliasghar Ahmad Kiadaliri, PhD, Department of Clinical Sciences Lund, Orthopedics, Clinical Epidemiology Unit, Lund University, Lund, Sweden. Alireza Ahmadi, PhD, Kermanshah University of Medical Sciences, Kermanshah, Iran. Kedir Yimam Ahmed, MPH, Debre Markos University, Debre Markos, Ethiopia. Muktar Beshir Ahmed, MPH, College of Health Sciences, Department of Epidemiology, ICT and e-Learning Coordinator, Jimma University, Jimma, Ethiopia. Faris Hasan Al Lami, PhD, Baghdad College of Medicine, Baghdad, Iraq. Khurshid Alam, PhD, Murdoch Childrens Research Institute, The University of Melbourne, Parkville, Victoria, Australia; The University of Melbourne, Melbourne, VIC, Australia; The University of Sydney, Sydney, NSW, Australia. Deena Alasfoor, MSc, Ministry of Health, Al Khuwair, Oman. Reza Alizadeh-Navaei, PhD, Gastrointestinal Cancer Research Center, Mazandaran University of Medical Sciences, Sari, Iran. Juma M Alkaabi, MD, College of Medicine and Health Sciences United Arab Emirates University, Al-Ain City, United Arab Emirates. Fatma Al-Maskari, $\mathrm{PhD}$, College of Medicine \& Health Sciences, United Arab Emirates; University, Al-Ain City, United Arab Emirates. Rajaa Al-Raddadi, $\mathrm{PhD}$, Joint Program of Family and Community Medicine, Jeddah, Saudi Arabia. Khalid A. Altirkawi, MD, King Saud University, Riyadh, Saudi Arabia. Nahla Anber, PhD, Mansoura University, Mansoura, Egypt. Hossein Ansari, PhD, Health Promotion Research Center, Department of Epidemiology and Biostatistics, Zahedan University of Medical Sciences, Zahedan, Iran. Hamid Asayesh, PhD, Department of Medical Emergency, School of Paramedic, Qom University of Medical Sciences, Qom, Iran. Rana Jawad Asghar, MD, South Asian Public Health Forum, Islamabad, Pakistan. Tesfay Mehari Atey, MS, Mekelle University, Mekelle, Ethiopia. Tadesse Awoke Ayele, MS, University of Gondar, Gondar, Ethiopia. Till Bärnighausen, MD, Department of Global Health and Population, Harvard T. H. Chan School of Public Health, Harvard University, Boston, MA, United States; Africa Health Research Institute, Mtubatuba, KwaZulu-Natal, South Africa; Institute of Public Health, Heidelberg University, Heidelberg, Germany. Umar Bacha, PhD, School of Health Sciences, University of Management and Technology, Lahore, Pakistan. Aleksandra Barac, PhD, Faculty of Medicine, University of Belgrade, Belgrade, Serbia. Suzanne L. Barker-Collo, $\mathrm{PhD}$, School of Psychology, University of Auckland, Auckland, New Zealand. Bernhard T. Baune, PhD, School of Medicine, University of Adelaide, Adelaide, South Australia, Australia. Shahrzad BazarganHejazi, PhD, College of Medicine, Charles R. Drew University of Medicine and Science, Los Angeles, CA, United States; David Geffen School of Medicine, University of California at Los Angeles, Los Angeles, CA, United States. Neeraj Bedi, MD, College of Public Health and Tropical Medicine, Jazan, Saudi Arabia. Isabela M Bensenor, $\mathrm{PhD}$, University of São Paulo, São Paulo, Brazil. Adugnaw Berhane, PhD, College of Health Sciences, Debre Berhan University, Debre Berhan, Ethiopia. Addisu Shunu Beyene, MPH, College of Health and Medical Science, Haramaya University, Harar, Ethiopia. Zulfiqar A. Bhutta, PhD, Centre of Excellence in Women and Child Health, Aga Khan University, Karachi, Pakistan; Centre for Global Child Health, The Hospital for Sick Children, Toronto, ON, Canada. Dube Jara Boneya, MPH, Department of Public Health, Debre Markos University, Debre Markos, Ethiopia. Rohan Borschmann, PhD, The University of Melbourne, Melbourne, VIC, Australia; Murdoch Childrens Research Institute, Melbourne, VIC, Australia. Nicholas J. K. Breitborde, $\mathrm{PhD}$, The Ohio State University, Columbus, $\mathrm{OH}$, United States. Zahid A. Butt, PhD, Al Shifa Trust Eye Hospital, Rawalpindi, Punjab, Pakistan. Ferrán Catalá-López, PhD, Department of Medicine, University of Valencia/INCLIVA Health Research Institute and CIBERSAM, Valencia, Spain; Clinical Epidemiology 
Program, Ottawa Hospital Research Institute, Ottawa, Canada. Liliana G. Ciobanu, MS, School of Medicine, University of Adelaide, Adelaide, South Australia, Australia. Hadi Danawi, PhD, Walden University, Minneapolis, Minnesota, United States. Amare Deribew, $\mathrm{PhD}$, Nuffield Department of Medicine, University of Oxford, Oxford, United Kingdom; KEMRI-Wellcome Trust Research Programme, Kilifi, Kenya. Samath D. Dharmaratne, MD, Department of Community Medicine, Faculty of Medicine, University of Peradeniya, Peradeniya, Sri Lanka. Kerrie E. Doyle, PhD, RMIT University, Bundoora, VIC, Australia; Australian National University, Canberra, ACT, Australia. Aman Yesuf Endries, MPH, Arba Minch University, Arba Minch, Ethiopia. Emerito Jose Aquino Faraon, MD, College of Public Health, University of the Philippines Manila, Manila, Philippines; Department of Health, Manila, Philippines. André Faro, PhD, Federal University of Sergipe, Aracaju, Brazil. Maryam S. Farvid, PhD, Department of Nutrition, Harvard T. H. Chan School of Public Health, Harvard University, Boston, MA, United States; Harvard/MGH Center on Genomics, Vulnerable Populations, and Health Disparities, Mongan Institute for Health Policy, Massachusetts General Hospital, Boston, MA, United States. Wubalem Fekadu, MS, Bahir Dar University, Bahir Dar, Ethiopia. Seyed-Mohammad Fereshtehnejad, PhD, Department of Neurobiology, Care Sciences and Society (NVS), Karolinska Institutet, Stockholm, Sweden. Florian Fischer, PhD, School of Public Health, Bielefeld University, Bielefeld, Germany. Tsegaye Tewelde Gebrehiwot, MPH, Jimma University, Jimma, Ethiopia. Ababi Zergaw Giref, $\mathrm{PhD}$, Addis Ababa University, Addis Ababa, Ethiopia. Melkamu Dedefo Gishu, MS, Haramaya University, Dire Dawa, Ethiopia; Kersa Health and Demographic Surveillance System, Harar, Ethiopia. Alessandra Carvalho Goulart, PhD, Center for Clinical and Epidemiological Research Center- Hospital Universitario-University of São Paulo, São Paulo, Brazil; Center of Check of Hospital Sirio Libanes, São Paulo, Brazil. Tesfa Dejenie Habtewold, MS, University of Groningen, Groningen, Netherlands; Debre Berhan University, Debre Berhan, Ethiopia. Randah Ribhi Hamadeh, DPhil, Arabian Gulf University, Manama, Bahrain. Mitiku Teshome Hambisa, MPH, College of Health and Medical Sciences, Haramaya University, Harar, Ethiopia. Samer Hamidi, DrPH, Hamdan Bin Mohammed Smart University, Dubai, United Arab Emirates. Josep Maria Haro, MD, Parc Sanitari Sant Joan de Déu - CIBERSAM, Sant Boi de Llobregat (Barcelona), Spain. Mohammad Sadegh Hassanvand, PhD, Center for Air Pollution Research, Institute for Environmental Research, Tehran University of Medical Sciences, Tehran, Iran. Nobuyuki Horita, MD, Department of Pulmonology, Yokohama City University Graduate School of Medicine, Yokohama, Kanagawa, Japan. Mohamed Hsairi, MD, Department of Epidemiology, Salah Azaiz Institute, Tunis, Tunisia. Hsiang Huang, MD, Cambridge Health Alliance, Cambridge, MA, United States. Abdullatif Husseini, $\mathrm{PhD}$, Institute of Community and Public Health, Birzeit University, Birzeit, Palestine. Mihajlo B. Jakovljevic, PhD, Faculty of Medical Sciences, University of Kragujevac, Kragujevac, Central Serbia Sumadija, Serbia; The Center for Health Trends and Forecasts, Institute for Health Metrics and Evaluation, University of Washington, Seattle, Washington, United States. Spencer Lewis James, MD, Denver Health/University of Colorado, Denver, CO, United States. Jost B. Jonas, MD, Department of Ophthalmology, Medical Faculty Mannheim, Ruprecht-Karls-University Heidelberg, Mannheim, Germany. Amir Kasaeian, PhD, Hematology-Oncology and Stem Cell Transplantation Research Center, Tehran University of Medical Sciences, Tehran, Iran; Endocrinology and Metabolism Population Sciences Institute, Tehran University of Medical Sciences, Tehran, Iran. Yousef Saleh Khader, ScD, Department of Community Medicine, Public Health and Family Medicine, Jordan University of Science and Technology, Irbid, Jordan. Ejaz Ahmad Khan, MD, Health Services Academy, Islamabad, Pakistan. Abdullah Tawfih Abdullah Khoja, MD, Mohammed Ibn Saudi University, Riyadh, Saudi Arabia.
Ardeshir Khosravi, PhD, Iranian Ministry of Health and Medical Education, Tehran, Iran, Non-communicable Diseases Research Center, Tehran University of Medical Sciences, Tehran, Iran. Jagdish Khubchandani, PhD, Department of Nutrition and Health Science, Ball State University, Muncie, Indiana, United States. Daniel Kim, DrPH, Department of Health Sciences, Northeastern University, Boston, Massachusetts, United States. Yun Jin Kim, PhD, Faculty of Chinese Medicine, Southern University College, Skudai, Malaysia. Yoshihiro Kokubo, PhD, Department of Preventive Cardiology, National Cerebral and Cardiovascular Center, Suita, Japan. Ai Koyanagi, MD, Research and Development Unit, Parc Sanitari Sant Joan de Deu (CIBERSAM), Barcelona, Spain. Barthelemy Kuate Defo, $\mathrm{PhD}$, Department of Social and Preventive Medicine, School of Public Health, University of Montreal, Montreal, Quebec, Canada; Department of Demography and Public Health Research Institute, University of Montreal, Montreal, Canada. Heidi J. Larson, PhD, Department of Infectious Disease Epidemiology, London School of Hygiene \& Tropical Medicine, London, United Kingdom; Institute for Health Metrics and Evaluation, University of Washington, Seattle, Washington, United States. Asma Abdul Latif, PhD, Department of Zoology, Lahore College for Women University, Lahore, Punjab, Pakistan. Paul H Lee, PhD, Hong Kong Polytechnic University, Hong Kong, China. Cheru Tesema Leshargie, MPH, Debre Markos University, Debre Markos, Ethiopia. Ricky Leung, PhD, State University of New York, Albany, Rensselaer, NY, United States. Loon-Tzian Lo, MD, UnionHealth Associates, LLC, St. Louis, MO, United States; Alton Mental Health Center, Alton, IL, United States. Raimundas Lunevicius, $\mathrm{PhD}$, Aintree University Hospital National Health Service Foundation Trust, Liverpool, United Kingdom; School of Medicine, University of Liverpool, Liverpool, United Kingdom. Hassan Magdy Abd El Razek, MBBCH, Mansoura Faculty of Medicine, Mansoura, Egypt. Mohammed Magdy Abd El Razek, MBBCH, Aswan University Hospital, Aswan Faculty of Medicine, Aswan, Egypt. Reza Majdzadeh, PhD, Knowledge Utilization Research Center and Community Based Participatory Research Center, Tehran University of Medical Sciences, Tehran, Iran. Azeem Majeed, MD, Department of Primary Care \& Public Health, Imperial College London, London, United Kingdom. Reza Malekzadeh, MD, Digestive Diseases Research Institute, Tehran University of Medical Sciences, Tehran, Iran; Digestive Diseases Research Institute, Tehran University of Medical Sciences, Tehran, Iran. Jose Martinez-Raga, PhD, Hospital Universitario Doctor Peset, Valencia, Spain; CEU Cardinal Herrera University, Moncada, Spain. Habibolah Masoudi Farid, MD, State Welfare Organisation, Tehran, Iran. Mohsen Mazidi, PhD, Key State Laboratory of Molecular Developmental Biology, Institute of Genetics and Developmental Biology, Chinese Academy of Sciences, Beijing, China. John J. McGrath, PhD, Queensland Centre for Mental Health Research, The Park Centre for Mental Health, Wacol, Queensland, Australia; Queensland Brain Institute, University of Queensland, St. Lucia, QLD, Australia, National Centre for RegisterBased Research, Aarhus School of Business and Social Sciences, Aarhus University, Aarhus, Denmark. Ziad A. Memish, MD, Saudi Ministry of Health, Riyadh, Saudi Arabia; College of Medicine, Alfaisal University, Riyadh, Saudi Arabia. Walter Mendoza, MD, United Nations Population Fund, Lima, Peru. Melkamu Merid Mengesha, MPH, College of Health and Medical Sciences, Haramaya University, Harar, Ethiopia. Mubarek Abera Mengistie, MS, Jimma University, Jimma, Ethiopia. George A. Mensah, MD, Center for Translation Research and Implementation Science, National Heart, Lung, and Blood Institute, National Institutes of Health, Bethesda, MD, United States. Haftay Berhane Mezgebe, MS, Mekelle University, Mekelle, Ethiopia. Ted R. Miller, PhD, Pacific Institute for Research \& Evaluation, Calverton, MD, United States, Centre for Population Health, Curtin University, Perth, WA, Australia. Philip B. Mitchell, MD, University of New South Wales, Kensington, New South Wales, Australia. Alireza Mohammadi, PhD, Neuroscience 
Research Center, Baqiyatallah University of Medical Science, Tehran, Iran. Shafiu Mohammed, PhD, Health Systems and Policy Research Unit, Ahmadu Bello University, Zaria, Nigeria; Institute of Public Health, Heidelberg University, Heidelberg, Germany. Carla Makhlouf Obermeyer, DSc, Center for Research on Population and Health, Faculty of Health Sciences, American University of Beirut, Beirut, Lebanon. Felix Akpojene Ogbo, MPH, Centre for Health Research, Western Sydney University, Sydney, New South Wales, Australia. Elizabeth Palomares Castillo, PhD, Ministry of Health, Mexico City, Mexico City, Mexico; Universidad Nacional Autónoma de México (UNAM), Mexico City, Mexico City, Mexico. Christina Papachristou, PhD, Charité University Medicine Berlin, Berlin, Germany. Scott B. Patten, PhD, Department of Community Health Sciences, University of Calgary, Calgary, Alberta, Canada. George C. Patton, MD, Murdoch Childrens Research Institute, Department of Paediatrics, University of Melbourne, Melbourne, Victoria, Australia. David M Pereira, PhD, REQUIMTE/LAQV, Laboratório de Farmacognosia, Departamento de Química, Faculdade de Farmácia, Universidade do Porto, Porto, Portugal. Aslam Pervaiz, MHA, Postgraduate Medical Institute, Lahore, Pakistan. Michael Robert Phillips, MD, Shanghai Jiao Tong University School of Medicine, Shanghai, China; Emory University, Atlanta, Georgia, United States. Farshad Pourmalek, PhD, University of British Columbia, Vancouver, British Columbia, Canada Mostafa Qorbani, PhD, Non-communicable Diseases Research Center, Alborz University of Medical Sciences, Karaj, Iran. Amir Radfar, MD, A T Still University, Kirksville, MO, United States. Anwar Rafay, MS, Contech International Health Consultants, Lahore, Pakistan, Contech School of Public Health, Lahore, Pakistan. Vafa Rahimi-Movaghar, MD, Sina Trauma and Surgery Research Center, Tehran University of Medical Sciences, Tehran, Iran. Rajesh Kumar Rai, MPH, Society for Health and Demographic Surveillance, Suri, India. David Laith Rawaf, MD, WHO Collaborating Centre, Imperial College London, London, United Kingdom; North Hampshire Hospitals, Basingstroke, United Kingdom; University College London Hospitals, London, United Kingdom. Salman Rawaf, MD, Imperial College London, London, United Kingdom. Amany $\mathrm{H}$. Refaat, PhD, Walden University, Minneapolis, MN, United States; Suez Canal University, Ismailia, Egypt. Satar Rezaei, PhD, School of Public Health, Kermanshah University of Medical Sciences, Kermanshah, Iran. Mohammad Sadegh Rezai, MD, Mazandaran University of Medical Sciences, Sari, Iran. Gholamreza Roshandel, $\mathrm{PhD}$, Golestan Research Center of Gastroenterology and Hepatology, Golestan University of Medical Sciences, Gorgan, Iran; Digestive Diseases Research Institute, Tehran University of Medical Sciences, Tehran, Iran. Mahdi Safdarian, MD, Sina Trauma \& Surgery Research Center, Tehran University of Medical Sciences, Tehran, Iran. Mahdi Safiabadi, MD, Student Research Committee, Baqiyatallah University of Medical Sciences, Tehran, Iran. Saeid Safiri, $\mathrm{PhD}$, Managerial Epidemiology Research Center, Department of Public Health, School of Nursing and Midwifery, Maragheh University of Medical Sciences, Maragheh, Iran. Rajesh Sagar, MD, All India Institute of Medical Sciences, New Delhi, India. Mohammad Ali Sahraian, MD, MS Research Center, Neuroscience Institute, Tehran University of Medical Sciences, Tehran, Iran. Payman Salamati, MD, Sina Trauma and Surgery Research Center, Tehran University of Medical Sciences, Tehran, Iran. Abdallah M. Samy, $\mathrm{PhD}$, Ain Shams University, Cairo, Egypt, Lawrence, Kansas, United States. Benn Sartorius, PhD, Public Health Medicine, School of Nursing and Public Health, University of KwaZulu-Natal, Durban, South Africa; UKZN Gastrointestinal Cancer Research Centre, South African Medical Research Council (SAMRC), Durban, South Africa. Mete I. Saylan, PhD, Bayer Turkey, Istanbul, Turkey. Soraya Seedat, $\mathrm{PhD}$, Stellenbosch University, Cape Town, South Africa. Sadaf G. Sepanlou, PhD, Digestive Diseases Research Institute, Tehran University of Medical Sciences, Tehran, Iran. Masood Ali Shaikh, MD, Independent Consultant, Karachi, Pakistan. Morteza
Shamsizadeh, MPH, Department of Medical Surgical Nursing, School of Nursing and Midwifery, Hamadan University of Medical Sciences, Hamadan, Iran. Diego Augusto Santos Silva, PhD, Federal University of Santa Catarina, Florianopolis, Brazil. Jasvinder A. Singh, MD, University of Alabama at Birmingham and Birmingham Veterans Affairs Medical Center, Birmingham, Alabama, United States. Badr H. A. Sobaih, MD, King Saud University, Riyadh, Saudi Arabia. Chandrashekhar T. Sreeramareddy, MD, Department of Community Medicine, International Medical University, Kuala Lumpur, Malaysia. Dan J. Stein, PhD, Department of Psychiatry, University of Cape Town, Cape Town, South Africa; South African Medical Research Council Unit on Anxiety \& Stress Disorders, Cape Town, South Africa. Rizwan Suliankatchi Abdulkader, MD, Ministry of Health, Kingdom of Saudi Arabia, Riyadh, Saudi Arabia. Bryan L. Sykes, $\mathrm{PhD}$, Departments of Criminology, Law \& Society, Sociology, and Public Health, University of California, Irvine, Irvine, CA, United States. Rafael Tabarés-Seisdedos, PhD, Department of Medicine, University of Valencia, INCLIVA Health Research Institute and CIBERSAM, Valencia, Spain. Muawiyyah Sufiyan, MD, Ahmadu Bello University, Zaria, Nigeria. Karen M. Tabb, PhD, School of Social Work, University of Illinois at Urbana-Champaign, Champaign, Illinois, United States. Arash Tehrani-Banihashemi, PhD, Preventive Medicine and Public Health Research Center, Iran University of Medical Sciences, Tehran, Iran. Mohamad-Hani Temsah, MD, King Saud University, Riyadh, Saudi Arabia. Abdullah Sulieman Terkawi, MD, Department of Anesthesiology, University of Virginia, Charlottesville, VA, United States; Department of Anesthesiology, King Fahad Medical City, Riyadh, Saudi Arabia, Outcomes Research Consortium, Cleveland Clinic, Cleveland, $\mathrm{OH}$, United States. Roman Topor-Madry, PhD, Institute of Public Health, Faculty of Health Sciences, Jagiellonian University Medical College, Kraków, Poland; Faculty of Health Sciences, Wroclaw Medical University, Wroclaw, Poland. Kingsley Nnanna Ukwaja, MD, Department of Internal Medicine, Federal Teaching Hospital, Abakaliki, Nigeria. Olalekan A. Uthman, PhD, Warwick Medical School, University of Warwick, Coventry, United Kingdom. Stein Emil Vollset, DrPH, Center for Disease Burden, Norwegian Institute of Public Health, Bergen, Norway; Department of Global Public Health and Primary Care, University of Bergen, Bergen, Norway, Institute for Health Metrics and Evaluation, University of Washington, Seattle, Washington, United States. Tolassa Wakayo, MS, Jimma University, Jimma, Ethiopia. Yuan-Pang Wang, PhD, University of São Paulo Medical School, São Paulo, Brazil. Andrea Werdecker, $\mathrm{PhD}$, Competence Center Mortality-Follow-Up of the German National Cohort, Federal Institute for Population Research, Wiesbaden, Germany. Ronny Westerman, PhD, Federal Institute for Population Research, Wiesbaden, Germany; German National Cohort Consortium, Heidelberg, Germany. Abdulhalik Workicho, MPH, Jimma University, Jimma, Ethiopia; Ghent University, Ghent, Belgium. Mohsen Yaghoubi, MSc, School of Public Health, University of Saskatchewan, Saskatoon, Saskatchewan, Canada. Hassen Hamid Yimam, MPH, Mizan Tepi University, Mizan Teferi, Ethiopia. Naohiro Yonemoto, MPH, Department of Biostatistics, School of Public Health, Kyoto University, Kyoto, Japan. Mustafa Z Younis, DrPH, Jackson State University, Jackson, MS, United States. Chuanhua Yu, PhD, Department of Epidemiology and Biostatistics, School of Public Health, Wuhan University, Wuhan, China, Global Health Institute, Wuhan University, Wuhan, China. Maysaa El Sayed Zaki, PhD, Faculty of Medicine, Mansoura University, Mansoura, Egypt. Aisha O. Jumaan, PhD, Independent Consultant, Seattle, Washington, United States. Theo Vos, PhD, Institute for Health Metrics and Evaluation, University of Washington, Seattle, Washington, United States. Simon I. Hay, DSc, Oxford Big Data Institute, Li Ka Shing Centre for Health Information and Discovery, University of Oxford, Oxford, United Kingdom; Institute for Health Metrics and Evaluation, University of Washington, Seattle, Washington, United 
States. Mohsen Naghavi, PhD, Institute for Health Metrics and Evaluation, University of Washington, Seattle, Washington, United States. Nicholas J. Kassebaum, MD, Institute for Health Metrics and Evaluation, University of Washington, Seattle, Washington, United States; Department of Anesthesiology \& Pain Medicine, Seattle Children's Hospital, Seattle, Washington, United States. Christopher J. L. Murray, DPhil, Institute for Health Metrics and Evaluation, University of Washington, Seattle, Washington, United States.

\section{Compliance with ethical standards}

This manuscript reflects original work that has not previously been published in whole or in part and is not under consideration elsewhere. All authors have read the manuscript and have agreed that the work is ready for submission and accept responsibility for its contents.The authors of this paper have complied with all ethical standards and do not have any conflicts of interest to disclose at the time of submission. The funding source played no role in the design of the study, the analysis and interpretation of data, and the writing of the paper. The study did not involve human participants and/or animals; therefore, no informed consent was needed.

Funding This research was funded by the Bill \& Melinda Gates Foundation.

Conflict of interest The authors declare that they have no conflicts of interest at this time.

Open Access This article is distributed under the terms of the Creative Commons Attribution 4.0 International License (http://crea tivecommons.org/licenses/by/4.0/), which permits unrestricted use, distribution, and reproduction in any medium, provided you give appropriate credit to the original author(s) and the source, provide a link to the Creative Commons license, and indicate if changes were made.

\section{References}

Abouzahir C, Wardlaw T (2001) Maternal mortality at the end of a decade: signs of progress? Bull World Health Org 79:561-573

Alvarez JL, Gil R, Hernández V, Gil A (2009) Factors associated with maternal mortality in Sub-Saharan Africa: an ecological study. BMC Public Health 9:462. doi:10.1186/1471-2458-9-462

Bayati M, Akbarian R, Kavosi Z (2013) Determinants of life expectancy in eastern mediterranean region: a health production function. Int J Health Policy Manag 1:57-61. doi:10.15171/ ijhpm.2013.09

Buor D, Bream K (2004) An analysis of the determinants of maternal mortality in sub-Saharan Africa. J Womens Health 13:926-938. doi:10.1089/jwh.2004.13.926

Campbell OMR, Graham WJ, Lancet Maternal Survival Series steering group (2006) Strategies for reducing maternal mortality: getting on with what works. Lancet Lond Engl 368:1284-1299. doi:10.1016/S0140-6736(06)69381-1

Hertz E, Hebert JR, Landon J (1994) Social and environmental factors and life expectancy, infant mortality, and maternal mortality rates: results of a cross-national comparison. Soc Sci Med 39:105-114. doi:10.1016/0277-9536(94)90170-8

Homaie Rad E, Vahedi S, Teimourizad A et al (2013) Comparison of the effects of public and private health expenditures on the health status: a panel data analysis in eastern mediterranean countries. Int J Health Policy Manag 1:163-167. doi:10.15171/ijhpm.2013. 29
Institute for Health Metrics and Evaluation (2016) Global health data exchange I GHDx. http://ghdx.healthdata.org/. Accessed 1 June 2017

Institute for Health Metrics and Evaluation (2017) Global burden of disease data visualization. https://vizhub.healthdata.org/gbd-com pare/. Accessed 13 July 2017

Kassebaum NJ, Bertozzi-Villa A, Coggeshall MS et al (2014) Global, regional, and national levels and causes of maternal mortality during 1990-2013: a systematic analysis for the Global Burden of Disease Study 2013. Lancet 384:980-1004. doi:10.1016/ S0140-6736(14)60696-6

Kassebaum NJ, Arora M, Barber RM et al (2016a) Global, regional, and national disability-adjusted life-years (DALYs) for 315 diseases and injuries and healthy life expectancy (HALE), 1990-2015: a systematic analysis for the Global Burden of Disease Study 2015. Lancet 388:1603-1658. doi:10.1016/ S0140-6736(16)31460-X

Kassebaum NJ, Barber RM, Bhutta ZA et al (2016b) Global, regional, and national levels of maternal mortality, 1990-2015: a systematic analysis for the Global Burden of Disease Study 2015. Lancet 388:1775-1812. doi:10.1016/S0140-6736(16)31470-2

Liang J, Zhu J, Dai L et al (2010) Maternal mortality in China, 1996-2005. Int J Gynecol Obstet 110:93-96. doi:10.1016/j.ijgo. 2010.03 .013

Lim SS, Dandona L, Hoisington JA et al (2010) India's Janani Suraksha Yojana, a conditional cash transfer programme to increase births in health facilities: an impact evaluation. Lancet 375:2009-2023. doi:10.1016/S0140-6736(10)60744-1

Midhet F, Becker S, Berendes HW (1998) Contextual determinants of maternal mortality in rural Pakistan. Soc Sci Med 46:1587-1598

Mokdad AH, Forouzanfar MH, Daoud F et al (2016) Health in times of uncertainty in the eastern Mediterranean region, 1990-2013: a systematic analysis for the Global Burden of Disease Study 2013. Lancet Glob Health 4:e704-713. doi:10.1016/S2214109X(16)30168-1

Muldoon KA, Galway LP, Nakajima M et al (2011) Health system determinants of infant, child and maternal mortality: a crosssectional study of UN member countries. Glob Health 7:42. doi:10.1186/1744-8603-7-42

Peterson HB, Haidar J, Merialdi M et al (2012) Preventing maternal and newborn deaths globally: using innovation and science to address challenges in implementing life-saving interventions. Obstet Gynecol 120:636-642. doi:10.1097/AOG.0b013e3182632cc1

Randive B, San Sebastian M, De Costa A, Lindholm L (2014) Inequalities in institutional delivery uptake and maternal mortality reduction in the context of cash incentive program, Janani Suraksha Yojana: results from nine states in India. Soc Sci Med 123:1-6. doi:10.1016/j.socscimed.2014.10.042

Ribeiro PS, Jacobsen KH, Mathers CD, Garcia-Moreno C (2008) Priorities for women's health from the Global Burden of Disease study. Int J Gynaecol Obstet Off Organ Int Fed Gynaecol Obstet 102:82-90. doi:10.1016/j.ijgo.2008.01.025

Ronsmans C, Graham WJ (2006) Maternal mortality: who, when, where, and why. Lancet 368:1189-1200. doi:10.1016/S01406736(06)69380-X

Rowe AK, de Savigny D, Lanata CF, Victora CG (2005) How can we achieve and maintain high-quality performance of health workers in low-resource settings? Lancet Lond Engl 366:1026-1035. doi:10.1016/S0140-6736(05)67028-6

Thaddeus S, Maine D (1994) Too far to walk: maternal mortality in context. Soc Sci Med 38:1091-1110

UNICEF (2016) Female genital mutilation/cutting: a global concern. https://www.unicef.org/media/files/FGMC_2016_bro chure_final_UNICEF_SPREAD.pdf. Accessed 13 July 2017 
United Nations (2015) Transforming our world: the 2030 agenda for sustainable development. https://sustainabledevelopment.un.org/ post2015/transformingourworld. Accessed 12 July 2017

Vos T, Allen C, Arora M et al (2016) Global, regional, and national incidence, prevalence, and years lived with disability for 310 diseases and injuries, 1990-2015: a systematic analysis for the Global Burden of Disease Study 2015. Lancet 388:1545-1602. doi:10.1016/S0140-6736(16)31678-6

Wang H, Naghavi M, Allen C et al (2016) Global, regional, and national life expectancy, all-cause mortality, and cause-specific mortality for 249 causes of death, 1980-2015: a systematic analysis for the Global Burden of Disease Study 2015. Lancet 388:1459-1544. doi:10.1016/S0140-6736(16)31012-1
Warren E, Post N, Hossain M et al (2015) Systematic review of the evidence on the effectiveness of sexual and reproductive health interventions in humanitarian crises. BMJ Open. doi:10.1136/ bmjopen-2015-008226

WHO (2017) High-quality healthcare needed for girls and women who have experienced female genital mutilation. http://www. who.int/reproductivehealth/topics/fgm/en/

WHO, UNICEF, UNFPA, World Bank (2007) Maternal mortality in 2005. http://www.who.int/whosis/mme_2005.pdf. Accessed 13 July 2017

World Health Organization (1992) International Statistical classification of diseases and related health problems. Tenth Revision 